TABLE I.-Results of Treatment of 875 Patients admitted with Prostatic Symptoms

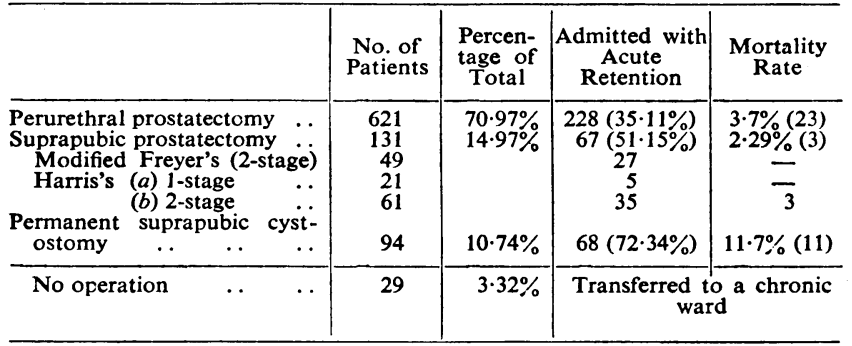

Mortality from all causes following prostatectomy is $3.4 \%$ in a consecutive series of 752 cases. Mortality of the last 439 perurethral prostatectomies in this series $=2.51 \%$ Of all prostatectomies $82.6 \%$ were performed perurethrally,
$17 \cdot 4 \%$ by various suprapubic methods. The oldest patient subjected to perurethrai operation was aged 90 .

TABLE II

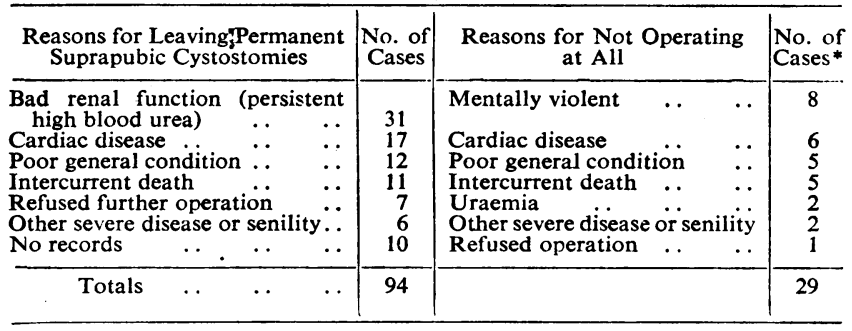

*All these were cases from the municipal hospital.

TABLE III.-Cause of Death after Prostatectomy

Cardiac failure, coronary thrombosis, and pulmonary embolism Uraemia

Clot retention :

Cerebral thrombosis

Fall in blood pressure after operation

Malignant endocarditis

Brain abscess following middle-ear infection
No records available ..

sent early to the surgeon. A clinical method of diagnosis, to prevent cases being missed, is described in detail. The indication for various types of operation is discussed. The perurethral operation is considered the method of choice. The paper is based upon a series of 875 consecutive cases, of which 752 were submitted to radical operation, with a mortality of $3.4 \%, 82.6 \%$ being treated by the perurethral method.

I wish to express my thanks to the sisters, nurses, and housesurgeons who have worked with me and without whose care and attention these results could not have been obtained. I also wish to thank my clinical and private assistant, Mr. Hadda, for the help he has given' me and for compiling the statistics.

\section{PARADOXICAL EMBOLISM}

\section{WITH REPORT OF A CASE DUE TO A VENTRICULAR SEPTUM DEFECT}

BY

\section{ALLAN BIRCH, M.D., F.R.C.P. Chase Farm Hospital, Enfield}

Various mechanisms have been advanced to explain how an embolus may avoid the lungs and pass from the systemic veins or right heart to the systemic arteries. The earliest explanation of the apparent contradiction to the normal path of emboli was that small ones could occasionally traverse the lung capillaries. This seems very unlikely. Experimental work suggests that even air cannot pass through the pulmonary circulation in harmful quantities. A patent foramen ovale has been present in fatal cases of paradoxical air embolism (Thomassen, 1938) and may have been present in non-fatal cases.

Since Cohnheim (1877) first traced the path of an embolus through an opening in the interauricular septum many such cases have been reported. An anatomically open interauricular septum may be functionally closed because the higher pressure in the left auricle ensures that it is closed by a valve on the left auricular side. Beattie (1925) suggested that this relation might be repressed by the lodging of a pulmonary embolus in the pulmonary artery which raises the pressure in the right auricle and at the same time lowers it in the left auricle because of diminished return from the lungs. A subsequent embolus arriving in the right auricle would be swept through to the left auricle and thence to the aorta. Thompson and Evans (1930) believed that at least one-third of the pulmonary arterial circulation had to be depleted before the right auricular pressure became greater than the left auricular pressure. That this mechanism is a possible one is suggested by the fact that in Wittig's (1927) series $50 \%$ of cases of paradoxical emboli were associated with antecedent pulmonary embolism. Some other explanation is necessary for those cases in which pulmonary embolism is absent or minimal. The simplest is that it is entirely fortuitous; a widely patent foramen ovale allows the embolus to pass without the help of pressure changes. Taylor (1934) suggested that shock from passage of a small embolus to the lung may bring about pressure changes which facilitate passage of a subsequent embolus through a patent foramen ovale. There is no experimental confirmation of this. Where the foramen is guarded by a valve Porter (1941) suggested that the embolus coils up over the tricuspid valve and obstructs it momentarily, so that the pressure rises in the right auricle and opens the foramen, allowing the embolus to pass through.

Paradoxical embolism may occur through a patent ventricular septum. If in addition the aorta arises from the right ventricle an easy route is provided for an embolus from a systemic vein to reach the aorta. Two such cases were described by Abbott and Beattie (1923) in which paradoxical embolism of the brain had arisen from septic foci in the arm and abdomen respectively. Only six examples of paradoxical embolism for which a ventricular septum defect was responsible have been reported. Louis (1826), in an article on communications between the right and left sides of the heart, described the case of a man aged 25 years in whom two areas of cerebral softening were found. The ventricular septum was defective at the base. No other cardiac anomaly was present. His description seems to indicate that the patient also had extensive pulmonary tuberculosis, and there is no clear evidence that the brain lesions were not tuberculomata. He did not draw any conclusions from these findings, but embolism was unknown in his day, being elucidated by Virchow only in 1845. Ballet (1880) collected a series of seven cases of congenital heart disease with cerebral abscess. In two of them a ventricular septum defect was the only anomaly. One was Louis's case and the other his own. This was a male of 15 years who died of a frontal abscess. The heart showed a ventricular septum defect with a malposed septum and an aplastic right ventricle. He assumed but did not explain the connexion between the septum defect and the cerebral abscess. Although the state of the peripheral veins is not given, the nasal fossae and cranial bones were normal, and paradoxical embolism seems to have been the probable cause. Stone (1881) reported the case of a female aged 19 years with a large ventricular septum defect and acute endocarditis of the stenosed conus of the right ventricle. She died of purulent meningitis and a cerebral abscess. No comment on the origin of the abscess was made. Hanna (1941), reviewing the 35 cases of paradoxical embolism reported up to 1941, found three (those of Louis, Ballet, and Stone) in which a ventricular septum defect was responsible, and added three of his own. In all six cases embolism was cerebral, and in none was the source of the embolus found.

In the case here reported the embolus consisted of vegetations from the pulmonary valve. It is suggested that they were large enough to lie over the opening in the septum and, when they broke off, to pass through it into the left ventricle. Pulmonary emboli were absent.

\section{Case Report}

A boy aged 11 had been blue and dyspnoeic on exertion since birth, and at the age of 6 was found to have congenital heart disease. At the age of 10 he had tonsillitis and cervical adenitis. A moderate amount of haematuria at the time was attributed to the nine days' sulphonamide treatment before admission to hospital, for no evidence of bacterial endocarditis was found. There was a very loud apical systolic bruit, and a basal to-and-fro bruit at the pulmonary area. He was readmitted at the age of 11 with pyrexia, sore throat, and pain in the right leg attributed to acute rheumatism. His temperature was $103^{\circ} \mathrm{F}$. It was found that the pain was due to arterial occlusion, and as similar signs were appearing in the left leg a diagnosis of embolism of both common iliac arteries from bacterial endocarditis was made. Haematuria was present, but urinary output 
was good. Streptococcus viridans grew on blood culture. He died four days later. At the necropsy a large embolus straddled the aortic bifurcation and completely occluded the right and a portion of the left common iliac artery. Recent infarcts were present in the left kidney and spleen. The mitral and aortic valves were normal. A large defect was present in the ventricular septum, but the auricular septum was normal. The cusps of the pulmonary valve could not be identified, and were replaced by a mass of friable vegetations. No infarcts were found in the lung.

\section{REFERENCES}

Abbott, M. E., and Beattie, W. W. (1923). Amer. J. med. Sci., 165, 636.

Ballet, G. (1880). Arch. gén. Méd., 145, 659.

Beattie, W. W. (1925). Bull. int. Ass. med. Museums, 11, 64.

Cohnheim, J. (1877). Vorlesungen über allgemeine Pathoiogie, 1, 134, Berlin

Hanna, R. (1941). Amer. J. Dis. Child., 62, 55s.

Louis, P. C. A. (1826). Recherches anatomico-pathologiques, p. 301, Obs. X, Paris. Porter, A. G. (1941). Lancet, 2, 634.

Stone, W. H. (1881). St. Thomas's Hosp. Rep., n.s. 11, 57.

Taylor, J. S. (1934). Bull. Ayer clin. Lab., 3, 39.

Thomassen, K. (1938). Norsk Mag. Laegevidensk., 99, 470.

Thompson, T., and Evans, W. (1930). Quart. J. Med., 23, 135

Wittig, M. (1927). Z. Kreislaufforsch., 19, 505, Dresden and Leipzig.

\section{ACTINOMYCOSIS SUCCESSFULLY TREATED WITH PENICILLIN \\ REPORT OF TWO CASES

$$
\text { BY }
$$

\section{A. J. C. HAMILTON, F.R.C.S.}

AND

\section{H. J. R. KIRKPATRICK, M.B., Ch.B., D.P.H.}

(Royal Northern Infirmary, Inverness)

Actinomycosis is not a common condition, and opportunities for observing the effects of its treatment with penicillin are consequently infrequent. Roberts et al. (1945) record two cases of thoracic actinomycosis. One of these patients died from Ps. pyocyanea pyaemia after a week's treatment with penicillin ; the other relapsed after one course of treatment $(1,800,000$ units administered systemically in 15 days), but recovered after a further course of treatment $(200,000$ units daily for 28 days). Hendrickson and Lehman (1945) record two cases of cervicofacial actinomycosis successfully treated with penicillin. In one case 2,000,000 units of the drug were administered in 16 days ; in the other $1,500,000$ units in 15 days. In a case treated by Macnab (1945) (daily intramuscular injections of 100,000 units for 10 days) penicillin had no apparent effect on the course of the disease.

We record below two cases of cervico-facial actinomycosis in which treatment with penicillin resulted in clinical cure. In one of these (Case 2) the history and physical signs pointed to the development of the lesion in the tonsillar lymph gland. There was no apparent actinomycotic lesion of the mouth or throat, but presumably lymphatic infection occurred through the tonsillar region. The occurrence of actinomycosis in lymph glands has been denied by some observers, and recently by Macnab (1945), but has been accepted by Cope (1938), who depicts actinomycotic lesions in photomicrographs of lymph glands.

\section{Case Histories}

Case 1.-A linotype operator aged 35 was admitted to hospital on May 15,1945 , with a large, brawny, smooth, painless, semifluctuant swelling situated over the inferior ramus of the left mandible. The swelling was ill defined, and extended upwards over the anterior border of the masseter muscle and downwards for a short distance into the submaxillary triangle. One enlarged lymph gland was felt at the anterior border of the sternomastoid. There was marked caries of the teeth. The lesion had apparently started 14 days before admission as a "gumboil " over the left lower jaw. Three teeth had been extracted on May 12. The lesion was incised and pus evacuated on the day of admission.

Case 2.-A railway porter aged 15 attended as an out-patient on May 31, 1945, with an enlarged right tonsillar gland, showing periadenitis and tenderness. He gave a history of a swelling in the right side of his neck a year before, which had soon disappeared. Nine weeks before admission he developed a sore throat, and the swelling in the neck reappeared and showed no tendency to diminish in size. Both tonsils were grossly enlarged and almost touching; the teeth were healthy, although some had been extracted a year and a half previously. He was referred to Dr. Seex, who removed his tonsils by dissection on June 4 . He was readmitted to hospital on June 14 with a brawny swelling of the neck, the inflammation now being definitely extracapsular. This was treated with ichthyoland-glycerin foments, and was incised on June 22 . Thick greenish pus was evacuated, and a rubber dam drain was left in the large abscess cavity.

Bacteriology.-The pus from both cases contained streptothrix filaments. These organisms failed to grow under aerobic conditions in primary cultures and in subcultures. In glucose-agar shakecultures there was no growth in the upper centimetre of the medium, and growth was maximal in a zone situated one centimetre below the surface of the medium. Growth occurred under anaerobic conditions but less readily than under micro-aerophilic conditions. Microscopical examination of the pus from Case 1 showed scanty Grampositive cocci as well as streptothrix filaments, but the cocci failed to grow in aerobic and anaerobic cultures, and were presumably non-viable. Only streptothrix filaments were noted in the pus from Case 2.

Dosage of Penicillin and Method of Administration.-In both cases penicillin was administered systemically by intramuscular injection During the first half of the course of treatment the dosage was 33,000 units every three hours; thereafter the dosage was reduced to 25,000 units every three hours. There was a gradual diminution in the size of the lesion, and treatment was continued until the discharge had ceased and the lesions had markedly diminished in size Case 1 received in all $5,800,000$ units of penicillin, and Case 2 $5,200,000$ units.

The patients were re-examined a month after discharge from hospital. In both there was slight thickening of the tissues at the site of the lesion but no evidence of an active inflammatory process.

\section{Discussion}

Different strains of Actinomyces bovis vary in their susceptibility to penicillin. Of five strains examined by Garrod (1944) and compared with the Oxford $\mathbf{H}$ strain of Staphylococcus aureus, two strains showed approximately the same degree of sensitivity as the standard staphylococcus; a third strain was eight times as resistant; a fourth strain four times as resistant and a fifth strain grew sparsely and atypically in 16 times the concentration of penicillin tolerated by the staphylococcus. The patient from whom this fifth strain was isolated was not treated with penicillin.

Fibrous-tissue formation is an outstanding characteristic of the pathology of actinomycosis-its amount and maturity varying with such factors as the age and site of the lesion, the resistance of the patient's tissues, and the virulence of the infecting organism. The amount of fibrous and granulation tissue, and its age, affect the vascularity of the lesion and the flow of blood through it, and consequently the permeation of the lesion by penicillin in the blood and the concentration of the drug attained in different parts of the lesion. Further, actinomyces may show a considerable degree of latency, and relapse may occur a long time after apparent cure (Cope, 1938).

Local treatment could not be applied in the two cases here described, and the relatively high dosage of penicillin administered systemically and the duration of the treatment were based on the response to treatment and on the above considerations, which seem to indicate the need for high dosage and a prolonged cGurse to ensure success in the treatment of this disease with penicillin.

The results obtained strikingly demonstrated the curative effect of penicillin, but more prolonged observation of the patients will be required to ensure that no viable actinomyces capable of proliferation remain embedded in the tissues.

\section{REFERENCES}

Cope, Z. (1938) Actinomycosis, Oxford University Press, London.

Cope, Z. (1938). Actinomycosis, Oxford University Press,

Garrod, L. P. (1944). British Medical Journal, 1, 528.

Roberts, J. E. H., Tubbs, O. S., and Bates, M. (1945). Lancet, 1, 40.

The first two of the lecture-demonstrations arranged by the British Medical Students' Association for its members in the London Region have now taken place. Mr. Claud Mullins opened the series with a talk on "Medicine and the Magistrates' Courts," and many students have since visited his court to see a demonstration of some of the points he made. Prof. E. Hindle gave an address on "The Prosectorium of the Zoo," and later conducted a party round the Zoological Gardens and the post-mortem department to demonstrate the investigations made into the physiology, anatomy, and pathology encountered among the animals. Details of future talks and visits (open to members of the Association only) may be obtained from the B.M.S.A. secretary at B.M.A. House, Tavistock Square, W.C.1. 